\title{
РАДИАЦИОННАЯ БЕЗОПАСНОСТЬ
}

\author{
DOI: http://dx.doi.org/10.22328/2079-5343-2018-9-1-178-184
}

\section{ВОЗМОЖНОСТИ ОБСЛЕДОВАНИЯ НОВОРОЖДЕННЫХ С ПОМОЩЬЮ НИЗКОДОЗОВОЙ МИКРОФОКУСНОЙ РЕНТГЕНОГРАФИИ}

${ }^{1}$ А. В. Алхазишвили, ${ }^{1}$ Л. Г. Константинова, ${ }^{2}$ В. Б. Бессонов, ${ }^{2}$ Ю. Н. Потрахов, ${ }^{2}$ Н. Н. Потрахов, ${ }^{1}$ Г. Е. Труфанов

${ }^{1}$ Национальный медицинский исследовательский центр им. В. А. Алмазова, Санкт-Петербург, Россия

${ }^{2}$ Санкт-Петербургский государственный электротехнический университет «ЛЭТИ» им. В. И. Ульянова (Ленина), Санкт-Петербург, Россия

\begin{abstract}
Новорожденные дети с различными патологиями нуждаются не только в первичном рентгенологическом исследовании, но и в неоднократном применении данного метода при динамическом наблюдении. Особенно это касается детей с изменениями в сердечно-сосудистой и дыхательной системах. Актуальной проблемой является повышение информативности традиционного рентгенологического исследования со снижением лучевой нагрузки на пациента без потери качества изображения
\end{abstract}

\section{THE POSSIBILITIES OF IMAGING NEWBORNS WITH LOW-} DOSE MICROFOCUS RADIOGRAPHY

${ }^{1}$ A. Alkhazishvili, ${ }^{1}$ L. Konstantinova, ${ }^{2} \mathrm{~V}$. Bessonov,

${ }^{2} Y u$. Potrakhov, ${ }^{2}$ N. Potrakhov, ${ }^{1}$ G. Trufanov

${ }^{1}$ Almazov National Medical Research Centre, St. Petersburg, Russia 2Electrotechnical University «LETI», St. Petersburg, Russia

Newborn children with various pathologies need not only a primary radiology study, but also repeated application of this imaging with dynamic observation. It mostly concerns the children with pathologies in the cardiovascular and respiratory systems. Increasing informative value of a traditional X-ray study with decreasing dosage of the $\mathrm{X}$-ray radiation for the patient without loss of image quality is nowadays one of the most actual subjects for research.

Цель исследования. В последние годы стремительно развивается современная технология низкодозовой микрофокусной рентгенографии. Положительные отзывы она уже приобрела в таких областях, как травматология и ортопедия, ревматология и стоматология. Основными ее преимуществами являются высокое качество изображений, хорошая резкость и контрастность, что несомненно важно для выявления мелкой, но в то же время значимой патологии, низкая лучевая нагрузка на пациента и персонал группы А и высокая мобильность, вследствие малого размера и небольшого веса оборудования. Эти факторы способствуют развитию микрофокусной рентгенографии и внедрению данного метода в педиатрии, использованию его в роддомах, перинатальных центрах, детских больницах, поликлиниках и в неприспособленных для исследований пространствах, в полевых или домашних условиях.

Материалы и методы. На базе перинатального центра ФГБУ «НМИЦ им. В. А. Алмазова» Минздрава России в 2017 г. обследованы 58 пациентов при помощи низкодозового микрофокусного аппарата «Пардус», разработанного СПбГЭТУ «ЛЭТИ». Выполнено 58 бэбиграмм, включающих визуализацию головы, шеи, верхних и нижних конечностей, органов грудной клетки, брюшной полости, малого таза. Методика исследований была отработана на препаратах тел новорожденных с различными ростовесовыми показателями, включая препарат тела мертворожденного недоношенного. Опытным путем определили, что наилучшее качество получения бэбиграмм достигается при напряжении $55 \mathrm{kB}, 0,15$ мА, диаметром фокусного пятна менее 0,2 мм и временем экспозиции 0,2 c.

Результаты. В зависимости от локализации процесса все дети были разделены на три группы. 1 -ю группу составил 21 ребенок с патологией сердечно-сосудистой системы, 2 -ю группу -23 ребенка с патологией органов грудной клетки, 3-ю группу - 14 детей с подозрением на сердечно-легочную патологию. Сердечно-сосудистая патология в основном определялась пороками сердца, такими как открытое овальное окно, открытый артериальный проток или декстрапозиция магистральных сосудов, которые могли сочетаться между собой. Со стороны органов грудной клетки самой распространенной патологией был респираторный дистресс-синдром. В брюшной полости наиболее часто исключали некротический энтероколит. В сочетанной патологии органов грудной клетки и брюшной полости определялись диафраг- мальные грыжи. При проведении сравнительного анализа с группой пациентов с идентичными росто-весовыми показателями (аппарат Siemens Mobilett XP) интерпретация и оценка тех же областей не вызвала дополнительных затруднений. Стоит отметить, что была в 2 раза снижена лучевая нагрузка на пациентов, улучшена мобильность оборудования и удобство его использования.

Заключение. Таким образом, низкодозовая микрофокусная рентгенография может использоваться как самостоятельная методика в педиатрии и имеет перед используемым палатным и стационарным оборудованием существенное преимущество, заключающееся в удобстве использования, мобильности и существенном снижении лучевой нагрузки.

\section{ЛИТЕРАTУPA/REFERENCES}

1. Микрофокусная рентгенография в клинической практике: учебное пособие / Н.Н. Потрахов, Г.Е. Труфанов, А.Ю. Васильев и др. СПб.: ЭЛБИСПб, 2012. 80 c. [Potrakov N., Trufanov G., Vasil'ev A. et al. Low-dose microfocus radiography in clinical practice: study guide. Saint Petersburg: Izdatel'stvo YELBI-SPb, 2012, 80 p. (In Russ.)].

2. Педиатрия: национальное руководство: в 2 т. М.: ГЭОТАР-Медиа, 2009 T. 1.1024 с. (Серия «Национальные руководства»). [Pediatry: national tutorial. Vol. 1. Moscow: Izdatel'stvo Gheotar-Media, 2009, 1024 p. (In Russ.)] Сведения об авторах:

Алхазищвили Александр Владимирович - ФГБУ «Национальный медицинский исследовательский центр им. В. А. Алмазова» Минздрава России; 197341, СанктПетербург, ул. Аккуратова, д. 2; e-mail: alkhazishvilialex@gmail.com;

Константинова Лариса Геннадиевна - кандидат медицинских наук, ФГБУ «Национальный медицинский исследовательский центр им. В.А. Алмазова» Минздрава России; 197341, Санкт-Петербург, ул. Аккуратова, д. 2; e-mail: fmrc@almazovcentre.ru; Бессонов Виктор Борисович - кандидат технических наук, доцент Санкт-Петербургского государственного электротехнического университета «ЛЭТИ» им. В.И. Ульянова (Ленина); 197376, Санкт-Петербург, ул. Профессора Попова, д. 5; e-mail: oso@etu.ru; Потрахов Юрий Николаевич -Санкт-Петербургский государственный электротехнический университет «ЛЭТИ» им. В.И. Ульянова (Ленина); 197376, СанктПетербург, ул. Профессора Попова, д. 5; e-mail: oso@etu.ru;

Потрахов Николай Николаевич - доктор технических наук, профессор Санкт-Петербургского государственного электротехнического университета «ЛЭТИ» им. В. И. Ульянова (Ленина); 197376, Санкт-Петербург, ул. Профессора Попова, д. 5; e-mail: oso@etu.ru; Труфанов Геннадий Евгеньевич - доктор медицинских наук, профессор ФГБУ

«Национальный медицинский исследовательский центр им. В. А. Алмазова» Минздрава России; 197341, Санкт-Петербург, ул. Аккуратова, д. 2; e-mail: fmrc@almazovcentre.ru.

ОСНОВНЫЕ ПРИЧИНЫ ПОВЫШЕННЫХ ДОЗ ОТ МЕДИЦИНСКОГО ОБЛУЧЕНИЯ ПРИ ПРОВЕДЕНИИ РЕНТГЕНОГРАФИЧЕСКИХ ИССЛЕДОВАНИЙ ${ }^{1}$ А. В. Водоватов, ${ }^{1}$ В. Ю. Голиков, ${ }^{2,3}$ И. Г. Камышанская ${ }^{1}$ Санкт-Петербургский научно-исследовательский институт радиационной гигиены им. проф. П. В. Рамзаева, Санкт-Петербург, Россия

${ }^{2}$ Санкт-Петербургский государственный университет, Россия

${ }^{3}$ Городская Мариинская больница, Санкт-Петербург, Россия

В данной работе рассматриваются основные причины, обусловливающие аномально высокие стандартные эффективные дозы облучения пациентов при проведении рентгенографических исследований на цифровых и аналоговых рентгеновских аппаратах. Результаты работы базируются на анализе данных, собранных в 6 регионах РФ за 2009-2015 гг.

\section{LEADING CAUSES OF ABNORMALLY HIGH PATIENT DOSES IN RADIOGRAPHY}

${ }^{1}$ A. V. Vodovatov, ${ }^{1}$ V. Yu. Golikov, ${ }^{2,3}$ I. G. Kamyshanskaya

${ }^{1}$ St. Petersburg Research Institute of Radiation Hygiene name after professor P. V. Ramzaev, St. Petersburg, Russia

2St. Petersburg State University, Department of Oncology, Russia ${ }^{3}$ Sity Mariinsky hospital, St. Petersburg, Russia

The current study was focused on the identification of leading causes of abnormally high patient doses from the conventional radiography for digital and ana- 
logue X-ray units. The results were based on the data collected in 6 Russian regions in 2009-2015.

Введение. Оптимизация защиты пациентов в лучевой диагностике представляет собой сложный многоуровневый процесс, включающий в себя следующие основные этапы: определение стандартных доз облучения пациентов при выбранных видах исследований; установление референтных диагностических уровней; выявление аппаратов с аномально высокими дозами и, наконец, устранение причин избыточного облучения пациентов [1]

Цель исследования. Анализ причин аномально высоких доз облучения пациентов при проведении рентгенографических исследований.

Материалы и методы. Исходные данные были собраны по 203 рентгеновским аппаратам (142 аналоговых, 61 цифровых) из 101 медицинской организации из 6 регионов Российской Федерации в период 2009-2015 гг. [2]. Параметры проведения исследований и эффективные дозы пациентов были определены для рентгенографических исследований черепа, органов грудной клетки, ребер, шейного, грудного и пояснично-крестцового отдела позвоночника, таза и брюшной полости. За аномально высокие дозы принимали значения стандартных эффективных доз, превышающие значения 75\% перцентилей распределений стандартных доз более чем на $30 \%$

Результаты. По результатам сбора и анализа данных выявлены наиболее распространенные причины аномально высоких доз облучения пациентов. Для аналоговых рентгеновских аппаратов к данным причинам относятся: использование синечувствительной рентгеновской пленки и рентгеновских кассет без усиливающих экранов; использование ручной проявки рентгеновской пленки с превышением срока эксплуатации реактивов для проявки, а также существенный износ рентгеновских трубок и генераторов. Данные факторы обусловливают проведение рентгенографических исследований с использованием низких значений анодного напряжения (до 70-80 кВ) и высоких значений экспозиции ( 100 мАс и выше).

В цифровой рентгенографии аномально высокие дозы облучения пациентов были обусловлены использованием протоколов исследований для аналоговых рентгеновских аппаратов (при переходе на цифровое оборудование без достаточной переподготовки персонала); выполнением исследований на высоких значениях экспозиции (100мАс и выше) для получения субъективно высокого диагностического качества изображений; низкой чувствительностью или отсутствием калибровки цифровых приемников рентгеновского изображения; а также неисправностями в системах автоматического контроля экспозиции

При работе нескольких рентгенлаборантов на одном рентгеновском аппарате используемые ими протоколы проведения рентгенографических исследований существенно отличались (различия в стандартных эффективных дозах вплоть до двух раз). Для цифровых рентгеновских аппаратов с телеуправляемым столом-штативом было характерно позиционирование пациентов в режиме рентгеноскопии со скоростью до 5 кадров в секунду. Эффективная доза от позиционирования превышала дозу за исследование вплоть до 2-3 раз.

Проведение мероприятий, направленных на устранение причин аномально высоких доз облучения пациентов, позволит существенно снизить уровни облучения пациентов и улучшить диагностическое качество рентгеновских снимков.

\section{ЛИТЕРАTУPA/REFERENCES}

1. Водоватов А.В. Практическая реализации концепции референтных диагностических уровней (РДУ) для оптимизации защиты пациентов при проведении стандартных рентгенографических исследований // Радиационная гигиена. 2017. T. 10, № 1. С. 47-55. [Vodovatov A.V. Practical implementation of the diagnostic reference levels concept for the common radiographic examinations. Radiatsionnaya Gygiena, 2017, Vol. 10 (1), pp. 47-55. (In Russ.)]. doi: 10.21514/1998-426X-2017-10-1-47-55

2. Vodovatov A.V., Balonov M.I., Golikov V.Yu. et al. Proposals for the establishment of national diagnostic reference levels for radiography for adult patients based on regional dose surveys in Russian Federation. Rad. Prot. Dosim., 2016, doi: 10.1093/rpd/ncw341.

\section{Сведения об авторах:}

Водоватов Александр Валерьевич - кандидат биологических наук; и. о. заведующего лабораторией радиационной гигиены медицинских организаций ФБУН «Санкт-Петербургский научно-исследовательский институт радиационной гигиены им. проф. П.В. Рамзаева» Федеральной службы по надзору в сфере защиты прав потребителей и благополучия человека; 197101, СанктПетербург, ул. Мира, д. 8; e-mail: vodovatoff@gmail.com;

Голиков Владислав Юрьевич - ведущий научный сотрудник лаборатории радиационной гигиены медицинских организаций ФБУН «Санкт-

Петербургский научно-исследовательский институт радиационной гигиены им. проф. П. В. Рамзаева» Федеральной службы по надзору в сфере защиты прав потребителей и благополучия человека; 197101, Санкт-Петербург, ул. Мира, д. 8; e-mail:sg235@rambler.ru;

Камышанская Ирина Григорьевна - кандидат медицинских наук, доцент, доцент кафедры онкологии ФГБОУ ВО «Санкт-Петербургский государственный университет», кафедра онкологии; 199034, Санкт-Петербург, 21 линия Васильевского острова, д. 8а; заведующая рентгеновским отделением СПб ГБУЗ «Городская Мариинская больница»; 191014, Санкт-Петербург,

Литейный проспект, д. 56; e-mail: irinaka@mail.ru.

\section{ОБЕСПЕЧЕНИЕ РАДИАЦИОННОЙ БЕЗОПАСНОСТИ В ОТДЕЛЕНИЯХ ЯДЕРНОЙ МЕДИЦИНЫ} И. А. Звонова

Санкт-Петербургский научно-исследовательский институт радиационной гигиены им. проф. П. В. Рамзаева, Санкт-Петербург, Россия

В докладе изложены основные требования обеспечения радиационной безопасности персонала и пациентов в отделениях ядерной медицины при использовании открытых радионуклидных источников для целей диагностики и терапии.

\section{RADIATION SAFETY ASSURANCE IN NUCLEAR MEDICINE DEPARTMENTS}

\author{
I. A. Zvonova
}

St. Petersburg Research Institute of Radiation Hygiene name after professor P. V. Ramzaev, St. Petersburg, Russia

The basic requirements for personnel and patients radiation safety ensuring in nuclear medicine departments using of unsealed radionuclide sources for diagnostic and therapeutic purposes are discussed.

Основное требование использования открытых радиоактивных источников (ОРнИ) в медицине состоит в непревышении основных переделов дозы у персонала (эффективная доза (ЭфД) - 20 м3/год в среднем за 5 лет, но не более 50 м3в/год, эквивалентные дозы (ЭкД) в хрусталике глаз - 150 мЗв/год; в коже, кистях рук и стопах 500 мЗв/год) и у населения: ЭфД - 1 мЗв/год в среднем за 5 лет, но не более 5 мЗв/год, ЭкД - 15 мЗв/год для хрусталика и 50 мЗв/год для кожи, кистей и стоп. В ядерной медицине (ЯМ) это достигается выполнением требований РБ, которые сформулированы в основных документах: НРБ-99/2009, ОСПОРБ-99/2010, СанПиН 2.6.1.328815, МУ 2.6.1.189-04 и ряде других методических документов.

Правильное распределение помещений подразделения ЯМ с группированием их на зоны работы с ОРнИ, пребывания пациентов и зону общих помещений препятствует распространению ОРнИ за пределы рабочей зоны и ограничивает неоправданное облучение персонала и пациентов. Қаждому помещению подразделения ЯМ присваивается класс работ с ОРнИ с учетом используемых радионуклидов и их активности на рабочем месте. Қаждому классу работ соответствует перечень требований РБ, изложенный в ОСПОРБ-99/2010, например, помещение 2 класса должно быть отделено от других помещений саншлюзом и недоступно для посещения пациентов, материалы стен и пола должны позволять дезактивацию и влажную уборку, толщина и материал стен и перекрытий должны обеспечивать защиту соседних помещений и территорий от максимально возможного излучения в рабочем помещении. Фасовка радиофармпрепаратов должна производиться в вытяжном шкафу, оборудованном радиационной защитой.

Персонал подразделения ЯМ должен использовать индивидуальные средства защиты: спецодежду, перчатки, рентгенозащитный фартук (для работы с ${ }^{99 \mathrm{~m}}$ Тс и радиоизотопами йода); переносить шприц с активностью в защитном контейнере, а при наполнении шприца и ведении РФП пациенту использовать защитный кожух на шприц из ванадиевого сплава. Организация работы с пациентами должна быть направлена на минимизацию времени контакта с пациентами с введенным РФП и увеличением расстояния от персонала до пациентов в период после введения РФП. 
Радиационная защита пациентов обеспечивается, прежде всего, обоснованностью назначения радионуклидного исследования, когда необходимая диагностическая информации не может быть получена другими не лучевыми методами. Администрация медицинской организации и персонал отделения ЯМ должны обеспечить оптимальные условия проведения исследования: техническую исправность, надлежащую настройку и калибровку аппаратуры, выбор оптимальной дозировки вводимого РФП, подготовку пациента к исследованию и рекомендации по его поведению во время и после сканирования Методические указания МУ 2.6.1.3151-13 определяют процедуру оценки и учета доз у пациентов при радионуклидной диагностике.

При проведении исследований детям вводимая активность рассчитывается исходя из массы тела ребенка. Радионуклидное исследование беременной женщине проводится в исключительных случаях, обосновывается путем взвешивания диагностической пользы для матери и потенциального вреда для будущего ребенка, выполняется с информированного согласия пациентки. Перед проведением исследования у пациентки необходимо выяснить, нет ли у нее беременности, не кормит ли она ребенка грудью. Грудное кормление после проведения исследования следует временно прервать, а при некоторых процедурах прекратить полностью.

В подразделениях ЯМ необходимо вести журналы учета движения и расходования радионуклидов от поступления активности до списания радиоактивных отходов после выдержки на распад. Также ведутся журналы введения РФП пациентам, учета генераторов радионуклидов, учета закрытых РИ, журналы инструктажа персонала по технике безопасности в части РБ и ряд других отчетных форм.

Дополнительные требования предъявляются к защите окружающей среды и населения при проведении радионуклидной терапии (РНД) Прежде всего, это наличие «активных» палат для изолированного содержания терапевтических пациентов с усиленной конструкционной защитой от излучения пациентов. В НРБ-99/2009 установлены критерии выписки пациентов после РНД. Например, для ${ }^{131}$ I это не более 400 МБк радионуклида в теле, а измеренная мощность эквивалентной дозы не более 20 мкЗв/ч на расстоянии 1 м от пациента. Важным отличием терапевтического отделения от диагностического является наличие спецканализации для сбора и выдержки на распад радиоактивных выделений пациентов.

Радиационный контроль является неотъемлемой частью системы РБ в ЯМ. Индивидуальный дозиметрический контроль персонала группь А посредством постоянного ношения индивидуальных термолюминесцентных дозиметров, дает информацию о реальных дозах сотрудников. Периодический радиационный контроль рабочих мест во время выполнения рабочих процедур, сравнение измерений с установленными контрольными уровнями служат для оценки состояния РБ в подразделении и выявления возможностей для его улучшения.

\section{ЛИТЕРАTУPA/REFERENCES}

1. Нормы радиационной безопасности (НРБ-99/2009). СанПиН 2.6.1.2523-09. [Norms of radiation safety (NRB-99/2009). SanPiN 2.6.1.2523-09. (In Russ.)].

2. Основные санитарные правила обеспечения радиационной безопасности (ОСПОРБ-99/2010). СП 2.6.1.2612-10, в редакции от 16.09.2013. [Basic sanitary rules for radiation safety assurance (OSPORB-99/2010). SP 2.6.1.2612-10, revised version of September 16, 2013. (In Russ.)].

3. Гигиенические требования по обеспечению радиационной безопасно сти при подготовке и проведении позитронно-эмиссионной томографии. СанПиН 2.6.1.3288-15. [Hygienic requirements on radiation safety assurance by the positron emission tomography preparation and implementation. SanPiN 2.6.1.3288-15. (In Russ.)].

4. Гигиенические требования по обеспечению радиационной безопасно сти при проведении радионуклидной диагностики с помощью радиофармпрепаратов. МУ 2.6.1.1892-04. [Hygienic requirements on radiation safety ensuring during radionuclide diagnostics using radiopharmaceuticals. Methodical guide. MУ 2.6.1.1892-04 (In Russ.)].

5. Оценка и учет эффективных доз у пациентов при проведении радионуклидных диагностических исследований. Методические указания МУ 2.6.1.3151-13. [Evaluation and recording of effective doses in patients with radionuclide diagnostic studies. Methodical guide, MY 2.6.1.3151-13. (In Russ.)] Сведения об авторе:

Звонова Ирина Александровна - доктор технических наук, главный научный сотрудник ФБУН «Санкт-Петербургский научно-исследовательский институт радиационной гигиены им. проф. П. В. Рамзаева» Федеральной службы по надзору в сфере защиты прав потребителей и благополучия человека; 197101, Санкт-Петербург, ул. Мира, д. 8; e-mail: ir_zv@bk.ru.

\section{К ПРОБЛЕМЕ ОБОСНОВАННОСТИ ПРОВЕДЕНИЯ РЕНТГЕНО- ЛОГИЧЕСКИХ ИССЛЕДОВАНИЙ В ЛУЧЕВОЙ ДИАГНОСТИКЕ}

\author{
C. А. Кальницкий
}

Санкт-Петербургский научно-исследовательский институт радиационной гигиены им. проф. П. В. Рамзаева, Санкт-Петербург, Россия

\section{DECISION OF PROBLEM JUSTIFICATION X-RAY EXAMINATIONS IN DIAGNOSTIC RADIOLOGY} S. A. Kalnitsky

St. Petersburg Research Institute of Radiation Hygiene name after professor P. V. Ramzaev, St. Petersburg, Russia

A criterion for the validity of justification $\mathrm{X}$-ray in diagnostic radiology is proposed. Its essence consists in comparing the preliminary direction of the attending physician and the final diagnosis of the radiologist. As a reasonableness it is suggested to use the sensitivity of the study. The implementation of the method should be used at the regional level, but not the specific doctor. It should be treated, not as a normative, but as an indicator of good practice in the type of diagnostic reference levels (DRL) in optimizing of radiation protection.

Введение. Рентгеновская диагностика является основным способом визуализации внутренних органов и тканей организма человека при обследовании пациентов по поводу заболеваемости. Ее масштабы огромны и постоянно увеличиваются и расширяются [1]. На каждого жителя приходится почти две рентгеновские процедуры (РП) в год, и наблюдается устойчивый рост их количества - на $30 \%$ за последние 10 лет при росте заболеваемости на $10 \%$ за этот же период [2]. Таким образом, рост числа РП опережает рост заболеваемости почти в три раза. При этом необходимо отметить, что на пациентов от проведения РП ложится большая лучевая нагрузка, которая особенно возрастает в последнее время при использовании современных методов визуализации, включая компьютерную томографию и интервенционные исследования [3, 4]. Вместе с тем принципы радиационной защиты требуют получения клинической информации в лучевой диагностике с минимальным облучением пациента [5]. Одним из основных методов радиационной защиты и снижения доз облучения пациентов, помимо оптимизации, является обоснование РП - исключение или сведение к минимуму случаев неоправданного проведения РП [6]. Это позволит, во-первых, обеспечить должную систему качества исследований, во-вторых, снизить лучевую нагрузку на пациентов и население и, в-третьих, уменьшить затраты здравоохранения.

Цель работы. Найти конкретное практическое решение проблемы обоснованности проведения РП.

Материалы и методы. Исследования проводились в рентгенодиагностических отделениях различных медицинских организаций Санкт-Петербурга.

Результаты. Анализ радиационно-гигиенической обстановки в здравоохранении показал, что в лучевой диагностике происходят два разнонаправленных процесса: увеличение количества РП и снижение дозы облучения пациентов. Первый является проявлением необоснованности РП, а второй свидетельствует, несмотря на это, об эффективности проведения оптимизации уровня облучения. Для выработки механизма обоснованности РП предложена чувствительность, которая определяет эффективность обследований пациентов. Чувствительность равна вкладу пациентов, у которых результат исследования является истинно положительным, от общего числа пациентов [7]. Чем выше чувствительность, тем выше эффективность обследования пациентов. По величине чувствительности можно судить об обоснованности РП. И наоборот, неэффективность РП определяется специфичностью, используемых РП. Специфичность определяется вкладом пациентов, у которых результат исследования является истинно отрицательным, от общего числа пациентов. Чем выше специфичность, тем ниже эффективность обследования пациентов.

Суть предлагаемого метода состоит в том, что сравниваются результаты предварительного направления лечащего врача и заключительного диагноза врача-рентгенолога при проведении РП. Обоснованным РП считается такое, которое подтверждает наличие болезни у пациента на основании совпадения направлений (предварительных диагнозов), поставленных лечащим врачом и подтвержденных врачом-рентгенологом. Обоснованность исследования можно определить НА: основании анализа данных РП, 
фиксированных в «Журнале учета (проведения) рентгенологических исследований», который имеется в каждом рентгенодиагностическом кабинете, «Амбулаторной карте пациента» или «Истории болезни».

Заключение. Предложен критерий обоснованности исследований пациентов в рентгенологии. В качестве обоснованности предложено использовать чувствительность исследования. Метод не требует дополнительного финансирования. Его суть состоит в сопоставлении предварительного направления лечащего врача и заключительного диагноза врача-рентгенолога. Формой реализации обоснованности может быть статистическая форма, например, № 30. Контроль обоснованности целесообразно возложить на Росздравнадзор. Его реализация должна использоваться на уровне региона (медицинской организации), но не конкретного врача. Возможна анонимность метода. К нему следует относиться, не как к нормативу, а как к показателю хорошей практики по типу референтных диагностических уровней при оптимизации радиационной защиты пациентов [8].

\section{ЛИТЕРАTУPA/REFERENCES}

1. Балонов М.И., Голиков В.Ю., Звонова И.А. и др. Современные уровни медицинского облучения в России. Радиационная гигиена. 2015. Т. 8, № 3 C. 67-79. [Balonov M.I., Golikov V.Yu., Zvonova I.A. et al. Sovremennye urovni medicinskogo oblucheniya v Rossii. Radiacionaya gigiena, 2015, Vol. 8, No. 3, pp. 67-79. (In Russ.)].

2. Здравоохранение в России. 2015: Стат. сб. Росстат. М., 2015. [Zdravoohranenie v Rossii. 2015: Stat. sb. Rosstat. Moscow, 2015 (In Russ.)]

3. Братилова А.А., Голиков В.Ю., Қальницкий С.А. Уровень облучения пациентов при проведении рентгеновской компьютерной томографии в медицинских организациях Санкт-Петербурга и Ленинградской области // Радиационная гигиена. 2014. T. 7, № 3. С. 33-38. [Bratilova A.A., Golikov V.Yu., Kal'nickij S.A. Uroven' oblucheniya pacientov pri provedenii rentgenovskoj komp'yuternoj tomografi v medicinskih organizaciyah Sankt-Peterburga i Leningradskoj oblasti. Radiacionnaya gigiena, 2014, Vol. 7, No. 3, pp. 33-38 (In Russ.)].

4. Голиков В.Ю., Сарычева С.С., Балонов М.И., Қальницкий С.А. Оценка доз облучения пациентов при проведении интервенционных рентгенологических исследований // Радиационная гигиена. 2009. Т. 2, № 3. С. 26-31 [Golikov V.Yu., Sarycheva S.S., Balonov M.I., Kal'nickij S.A. Ocenka doz oblucheniya pacientov pri provedenii intervencionnyh rentgenologicheskih issledovanij. Radiacionnaya gigiena, 2009, Vol. 2, No. 3, pp. 26-31 (In Russ.)].

5. МАГАТЭ. Радиологическая защита при медицинском облучении ионизирующим излучением. Руководство по безопасности RS-G-1.5, МАГАТЭ, Вена, 2004. [MAGATEH. Radiologicheskaya zashchita pri medicinskom obluchenii ioniziruyushchim izlucheniem. Rukovodstvo po bezopasnosti RSG-1.5, MAGATEH, Vena, 2004 (In Russ.)].

6. IAEA. Justification of medical exposure in diagnostic imaging. Proceedings series. International Atomic Energy Agency. Vienna, 2011.

7. Рациональный подход к рентгенодиагностическим исследованиям. Доклад 689, ВО3. Женева, 1987. [Racional'nyj podhod k rentgenodiagnostic heskim issledovaniyam. Doklad 689, VOZ. Zheneva, 1987 (In Russ.)].

8. МКРЗ. Радиационная защита в медицине: Публикация 105 МКРЗ. СПб. НИИРГ, 2011. [MKRZ. Radiacionnaya zashchita v medicine: Publikaciya 105 MKRZ. Saint Petersburg: Izdatel'stvo NIIRG, 2011. (In Russ.)].

\section{Сведения об авторе:}

Кальницкий Сергей Анатольевич - старший научный сотрудник, ведущий научный сотрудник ФБУН «Санкт-Петербургский научно-исследовательский институт радиационной гигиены им. проф. П. В. Рамзаева»; 197101, СанктПетербург, ул. Мира, д. 8; e-mail: s.kalnitsky@niirg.ru.

\section{ОБЛУЧЕНИЕ МОЛОЧНОЙ ЖЕЛЕЗЫ У ЖЕНЩИН ПРИ РЕНТГЕНОЛОГИЧЕСКИХ ИССЛЕДОВАНИЯХ}

\author{
С. А. Кальницкий
}

Санкт-Петербургский научно-исследовательский институт радиационной гигиены им. проф. П. В. Рамзаева, Санкт-Петербург, Россия

\section{IRRADIATION OF THE BREAST IN WOMEN DURING X-RAY EXAMINATIONS}

S. A. Kalnitsky

St. Petersburg Research Institute of Radiation Hygiene name after professor P. V. Ramzaev, St. Petersburg, Russia

The modern level of breast irradiation in woman with various $\mathrm{X}$-ray examinations was analyzed. It is shown that the main radiation load on a woman's breast is formed not at the expense of mammography, but in other types of studies in which the breast gets into the field of irradiation. This mainly applies to lung and spine examinations. The high frequency of their conduct, especially of the lungs, increases the radiation impact on the patient, thus putting forward certain radiation protection requirements for breast during their conduct. It is suggested to consider the potential variants of radiation protection of breast in woman at different $\mathrm{X}$-ray examinations.

Введение. В настоящее время рак молочной железы (МЖ) у женщин занимает первое место среди всех онкологических заболеваний [1]. В этом аспекте проводится его профилактика, сопровождающаяся облучением МЖ. В то же время, согласно публикации 103 МКРЗ 2007 г., МЖ впервые была отнесена к наиболее радиочувствительным органам [2], то есть при облучении она подвергается максимальному риску. Это означает, что в медицинской рентгеновской диагностике МЖ в первую очередь нуждается в радиационной защите (как ранее гонады, которые тем же документом были отнесены к менее радиочувствительным органам). До настоящего времени такой постановки вопроса не существовало, поскольку МЖ не относилась к наиболее радиочувствительным органам [3]. Современное отечественное регулирование отстает от мирового, поэтому данный вопрос до сих пор был не актуален [4]. Однако, ввиду подготовки новой редакции Норм радиационной безопасности (НРБ), целесообразно предварительно рассмотреть данный вопрос как перспективный и необходимый аспект радиационной защиты.

Цель работы. Анализ современного уровня рентгенодиагностического облучения МЖ у женщин с целью разработки эффективных мер радиационной защиты в соответствии с современными радиобиологическими сведениями.

Материалы и методы. Работа была выполнена в рентгенодиагностических отделениях медицинских организаций Санкт-Петербурга. Определялись эквивалентные дозы на МЖ и эффективные дозы у женщин при различных рентгенологических исследованиях (РЛИ), включая маммографию.

Результаты. В ходе исследований определено, что МЖ, находясь практически в центре туловища женщины, при большинстве РЛИ попадает либо в прямой пучок излучения (исследования костей скелета), либо в рассеянное излучение при экранировании туловищем, например, в исследованиях органов грудной клетки. Во всех случаях МЖ в той или иной степени подвергается облучению. Среди различных локализаций обследования пациентов и облучения МЖ можно выделить основные: исследования скелета (в основном позвоночника), различные виды исследования легких (рентгеноскопия, рентгенография и флюорография), а также исследования желудочно-кишечного тракта. Средняя эквивалентная доза облучения МЖ пациенток от различных РЛИ равняется 0,2 мЗв, при снимках легких - 0,1 мЗв, при проведении маммографии - 1,0 мЗв за снимок. Для сравнения маммография формирует у женщины эффективную дозу, равную 0,1 мЗв при пленочном методе (0,05 мЗв - при цифровом) [5].

Максимальное облучение МЖ получает при нахождении их в прямом пучке излучения, тем не менее нельзя не учитывать дозы, полученные в рассеянном излучении. Вышесказанное свидетельствует о необходимости защиты МЖ по мере возможности при проведении РЛИ, включая исследования позвоночника, дозы которого являются весьма существенными (для шейных позвонков эффективная доза равняется 0,15 м3в, грудных - 0,40 мЗв, поясничных - 0,70 м3в) [5]. Прямая защита возможна в первую очередь при использовании боковых проекций.

Заключение. Проанализирован современный уровень облучения МЖ при различных РЛИ. Показано, что лучевая нагрузка на МЖ женщины формируется не только за счет маммографии, но и при других видах исследований, в которых МЖ попадает в поле облучения. В основном это касается обследований легких и позвоночника. Большая частота их проведения, особенно легких, увеличивает радиационное воздействие на пациентку, чем выдвигает определенные требования радиационной защиты МЖ при проведении РЛИ. Необходимо рассмотреть все возможные варианты защиты.

\section{ЛИТЕРАTУPA/REFERENCES}

1. Здравоохранение в России. 2015: Стат. сб. Росстат. М., 2015. [Zdravoohranenie v Rossii. 2015: Stat. sb. Rosstat. Moscow, 2015 (In Russ.)].

2. Публикация 103 Международной комиссии по радиационой защите (МКР3) / пер. с англ.; под общ. ред. М. Ф. Қиселева, Н. К. Шандалы. М.: Алана, 2009. [Publikaciya 103 Mezhdunarodnoj komissii po radiacionnoj 
zashchite (MKRZ): per. s angl.; pod obshchej red. M. F. Kiseleva, N. K. Shandaly. Moscow: Izdatel'stvo Alana, 2009. (In Russ.)].

3. Рекомендации международной комиссии по радиологической защите 1990 года. Публикация 50 МКР3. М.: Энергоатомиздат, 1994. [Rekomendaci mezhdunarodnoj komissii po radiologicheskoj zashchite 1990 goda Publikaciya 50 MKRZ. Moscow: Izdatel'stvo Ehnergoatomizdat, 1994 (In Russ.)]

4. СанПиН 2.6.12523-09 «Нормы радиационной безопасности НРБ99/2009». [SanPiN 2.6.12523-09 «Normy radiacionnoj bezopasnosti NRB99/2009». (In Russ.)]

5. Заполнение формы федерального государственного статистического наблюдения 3-ДОЗ. Методические рекомендации (проект). [Zapolnenie formy federal'nogo gosudarstvennogo statisticheskogo nablyudeniya 3-DOZ. Metodicheskie rekomendacii (proekt) (In Russ.)].

Сведения об авторе:

Кальнищкий Сергей Анатольевич - старший научный сотрудник, ведущий научный сотрудник ФБУН «Санкт-Петербургский научно-исследовательский институт радиационной гигиены им. проф. П. В. Рамзаева»; 197101, СанктПетербург, ул. Мира, д. 8; e-mail: s.kalnitsky@ niirg.ru.

\section{УРОВЕНЬ МЕДИЦИНСКОГО ОБЛУЧЕНИЯ ПАЦИЕНТОВ В ЛУЧЕВОЙ ДИАГНОСТИКЕ}

С. А. Кальницкий

Санкт-Петербургский научно-исследовательский институт радиационной гигиены им. проф. П. В. Рамзаева, Санкт-Петербург, Россия

\section{LEVEL OF MEDICAL EXPOSURE OF PATIENTS IN DIAGNOSTIC RADIOLOGY AND NUCLEAR MEDICINE IN RUSSIA}

\author{
S. A. Kalnitsky
}

St. Petersburg Research Institute of Radiation Hygiene name after professor P. V. Ramzaev, St. Petersburg, Russia

The levels of medical exposure of patients in different regions of Russia for a long time have been determined. A significant volume and structure of research is shown. Individual and collective doses of patients are given. The important role of radiation protection is determined.

Введение. Медицинское облучение (МО) является важным фактором облучения всего населения [1]. Во всем мире его уровень увеличивается, особенно заметно в экономически развитых странах и в последнее время, несмотря на принятые меры по ограничению МО в рамках процесса оптимизации радиационной защиты (Р3) в виде использования референтных диагностических уровней (РДУ) [2, 3] Данная концепция принята на вооружение и в РФ, однако до сих пор она не нашла практического применения. В РФ МО до последнего времени снижалось благодаря ранее принятым мерам Р3, однако в последние годы наметилась тенденция его увеличения [4] Представлялось целесообразным определить и проанализировать уровень МО и проследить его динамику. Причиной такого внимания является широкое внедрение новых инновационных методов радиационных (рентгенорадиологических) процедур (РРП), сопровождающихся высокими дозами облучения пациентов [5].

Цель работы. Определить количество РРП, уровень МО и их динамику в РФ. Проанализировать происходящие процессы с точки зрения Р3.

Материалы и методы. Исследования проводились на протяжении последнего десятилетия в рентгенорадиологических отделениях многочисленных медицинских организаций нескольких регионов РФ и касались рентгеновских исследований, компьютерной томографии, интервенционной радиологии и радионуклидной диагностики.

Результаты. Показано, что в последнее время в отечественной лучевой диагностике прослеживается две тенденции МО: индивидуальные дозы у пациентов снижаются за счет совершенствования аппаратуры и оборудования и увеличиваются за счет внедрения принципиально новых высокоинформативных технологий, в основном компьютерных, сопровождающихся повышенными индивидуальными дозами облучения пациентов (KT, интервенционные исследования и др.). В последние годы преобладающей стала вторая тенденция. Эффективные дозы при проведении данных РРП достигают десятков миллизиверт, а доза в коже может вызывать лучевые поражения. Более того, наметилось комплексное использование таких методов, например, ПЭТ/КТ и др., сопровождающихся еще более высокими лучевыми нагрузками. При этом наблюдается тенденция, при которой дозы у пациентов для одних и тех же видов исследований значительно отличаются в разных медицинских организациях (до 100 раз), что указывает на отсутствие оптимизации и значительные резервы для их снижения.

Суммарное количество всех диагностических РРП в России в 2016 г. достигло 275 млн. Это означает, что частота РРП равняется $1877 \%$, то есть на каждого жителя приходится почти две процедуры в год и наблюдается их устойчивый рост, в основном за счет КТ. Тем не менее в структуре РРП преобладают традиционные РРП: рентгенография $(64,4 \%$ всех РРП) и флюорография $(30,9 \%)$. Вклад КТ составляет лишь $3,4 \%$, но обусловливает почти половину всей лучевой нагрузки. В результате средняя доза за процедуру составляет 0,27 мЗв, а средняя доза облучения населения $-0,51$ мЗв на человека. Максимальные индивидуальные дозы наблюдаются при специальных исследованиях - 5,0 м3в, КТ - 3,9 мЗв, рентгеноскопии 2,5 мЗв и в радионуклидной диагностике 3,4 м3в, минимальные дозы: при флюорографии - 0,07 м3в и рентгенографии - 0,10 м3в

Заключение. Доза, получаемая пациентами при РРП, определяется как характеристиками рентгенорадиологического оборудования и используемыми методиками (рентгеновского аппарата, рентгеновской пленки, введенной активности радионуклида и др.), так и квалификацией персонала, обслуживающего данное оборудование. В этих условиях необходимо совершенствование РЗ пациентов для недопущения значительного увеличения уровня МО населения, в том числе расширение образовательной работы, включая радиационную безопасность, на уровне базового образования, профессиональной переподготовки и деятельности профессиональных (рентгенорадиологических) сообществ.

\section{ЛИТЕРАTУPA/REFERENCES}

1. UNSCEAR. Sources and Effects of Ionising Radiation. UNSCEAR 2008 Report to the General Assembly with Scientific Annexes. Vol. I, Annex A. New York, UN, 2010

2. МАГАТЭ. Радиологическая защита при медицинском облучении ионизирующим излучением. Руководство по безопасности RS-G-1.5, Вена, МАГАТЭ, 2004. [MAGATEH. Radiologicheskaya zashchita pri medicinskom obluchenii ioniziruyushchim izlucheniem. Rukovodstvo po bezopasnosti RSG-1.5, Vena, MAGATEH, 2004. (In Russ.)].

3. МКР3. Радиационная защита в медищине: Публикация 105 МКР3. СПб.: НИИРГ, 2011. [MKRZ. Radiacionnaya zashchita v medicine: Publikaciya 105 MKRZ. Saint Petersburg: Izdatel'stvo NIIRG, 2011. (In Russ.)].

4. Дозы облучения населения Российской Федеращии: серия информационных сборников. 2001-2016 гг. [Dozy oblucheniya naseleniya Rossijskoj Federacii: seriya informacionnyh sbornikov. 2001-2016 gg. (In Russ.)].

5. Балонов М.И., Голиков В.Ю., Звонова И.А. и др. Современные уровни медищинского облучения в России // Радиационная гигиена. 2015. Т. 8, № 3. C. 67-79. [Balonov M.I., Golikov V.Yu., Zvonova I.A. et al. Sovremennye urovni medicinskogo oblucheniya v Rossii. Radiacionnaya gigiena, 2015, Vol. 8, No. 3, pp. 67-79 (In Russ.)].

Сведения об авторе:

Кальницкий Сергей Анатольевич - старший научный сотрудник, ведущий научный сотрудник ФБУН «Санкт-Петербургский научно-исследовательский институт радиационной гигиены им. проф. П. В. Рамзаева»; 197101, СанктПетербург, ул. Мира, д. 8; e-mail: s.kalnitsky@niirg.ru.

Открыта подписка на 2-е полугодие 2018 года. Подписные индексы:

Агентство «Роспечать» 57991 000 «Агентство „Книга-Сервис"» 42177

\section{ОПТИМИЗАЦИЯ ПРОВЕДЕНИЯ ПЭТ-КТ-ИССЛЕДОВАНИЙ}

\section{Л. А. Чипига}

Санкт-Петербургский научно-исследовательский институт радиационной гигиены им. проф. П. В. Рамзаева, Санкт-Петербург, Россия

Национальный медицинский исследовательский центр им. В. А. Алмазова, Санкт-Петербург, Россия

Целью данной работы являлась оценка уровней облучения пациентов и возможных путей оптимизации позитронной эмиссионной томографии (ПЭТ) в России по результатам сбора данных в 12 регионах Российской Федерации. Сравнение результатов с зарубежными данными показало, что дозы в России выше, чем в других странах. Перспективна оптимизация радиационной защиты пациентов за счет обоснования назначений многофазного ҚТ сканирования и использования низкодозовых КТ протоколов 


\section{OPTIMIZATION OF PET-CT RESEARCH}

L. A. Chipiga

St. Petersburg Research Institute of Radiation Hygiene name after professor P. V. Ramzaev, St. Petersburg, Russia

National Almazov Medical Research Centre, St. Petersburg, Russia

The aim of the study was to estimate a patient dose in and to suggest possible methods for optimization in positron emission tomography (PET) in Russia. A dose survey of PET examinations was performed in 12 regions. The comparison with similar published data indicate that the patient doses in Russia are higher than in other countries. Low-dose CT protocols and justification of multiphase CT protocols should be considered for future optimization and radiation protection of patients.

Цель исследования. Оценить уровни облучения пациентов и возможные пути оптимизации ПЭТ-КТ-исследований в России.

Материалы и методы. В 2012-2017 гг. обследовано 27 аппаратов в 19 отделениях ПЭТ-диагностики в 12 регионах России: Санкт-Петербург, Москва, Белгородская, Курская, Липецкая, Орловская, Свердловская, Тамбовская, Тюменская области, республики Башкортостан и Татарстан, Приморский край. В каждом отделении собиралась информация о видах исследований, применяемых радиофармпрепаратах (РФП) и активностях, вводимых стандартному пациенту, а также параметрах протоколов КТ-сканирования и дозах пациентов. Эффективные дозы, полученные пациентами за одно ПЭТ/КТ-исследование, определялись как сумма доз внутреннего облучения от введенного РФП [1] и внешнего рентгеновского облучения при КТ-сканировании [2, 3].

Результаты. Согласно полученным данным, наиболее распространенным ПЭТ-исследованием в России является исследование всего тела с ${ }^{18} \mathrm{~F}$-фтордезоксиглюкозой для диагностики онкологических заболеваний. Дозы облучения пациентов от ПЭТ/КТ-исследований лежат в диапазоне 3-40 мЗв. Наибольшие дозы получают пациенть при исследовании всего тела - около 17 мЗв, при этом КТ-сканирование вносит от $65 \%$ до 95\% в дозу облучения пациента. При дополнительных многофазных КТ-сканированиях с введением контрастного вещества доза может увеличиваться до 25-30 м3в.

Для оптимизации проведения ПЭТ-исследований, сравнения результатов, полученных в разных отделениях ПЭТ-диагностики, и повышения диагностической ценности данного метода в России необходима стандартизация используемых протоколов сбора и обработки данных, а также получение количественных критериев для сравнения результатов, полученных на томографах разных моделей. В связи с этим становится вопрос о разработке методики оценки качества и сравнительного анализа технических параметров ПЭТ-изображений

Для сравнения протоколов ПЭТ-исследований и определения критериев их оценки необходимы анализ и контроль количественных результатов ПЭТ-сканирования при использовании разных протоколов. Для снижения ошибок данного метода диагностики в России и стандартизации проведения ПЭТ-исследований целесообразно разработать и внедрить методику контроля параметров проведения исследования, нацеленную на оптимизацию диагностического качества изображения. Қак показывает зарубежный опыт, данное решение позволит улучшить точность количественной оценки распределения РФП путем контроля качества ПЭТ изображения. Оптимизация радиационной защиты пациентов возможна за счет использования низкодозового режима КТ сканирования вместо диагностического, создания специальных протоколов для отдельных групп пациентов, корректного применения системы автоматической модуляции силы тока и современных методов реконструкции изображения; обучения медицинского персонала техническим приемам снижения дозы и знаниям в области радиационной безопасности.

\section{ЛИТЕРАTУPA/REFERENCES}

1. МУ 2.6.1.3151-13 «Оценка и учет эффективных доз у пациентов при про ведении радионуклидных диагностических исследований». [Methodical gui dance 2.6.1.3151-13. Assessment and accounting of effective dose to the patients undergoing nuclear medicine diagnostic examinations (In Russ.)].

2. МУ 2.6.1.2944-11 «Контроль эффективных доз облучения пациентов при проведении медицинских рентгенологических исследований». [Methodical guidance 2.6.1.2944-11. Assessment of effective dose to the patients undergoing $X$-ray examinations. (In Russ.)].

3. Чипига Л.А., Голиков В.Ю., Шлеенкова Е.Н., Поздняков А.В. Оценка коэффициентов перехода от произведения дозы на длину сканирова ния к эффективной дозе для КТ всего тела путем фантомных экспери ментов // Медицинская физика, 2016. Т. 4. С. 55-62. [Chipiga L. Golikov V., Shleenkova E., Pozdnyakov A. Estimation of the conversion coefficients from dose length product to effective dose from whole body computed tomography examination using anthropomorphic phantoms. Medical physics, 2016, Vol. 4, pp. 55-62 (In Russ.)].

\section{Сведения об авторе:}

Чипига Лариса Александровна - младший научный сотрудник лаборатории радиационной гигиены медицинских организаций ФГУН «Санкт-

Петербургский научно-исследовательский институт радиационной гигиены им. проф. П. В. Рамзаева» Федеральной службы по надзору в сфере защиты прав потребителей и благополучия человека; 197101, Санкт-Петербург, ул. Мира, д. 8; медицинский физик, ПЭТ-центр, ФГБУ «Национальный медицинский исследовательский центр им. В. А. Алмазова» Минздрава России; 197341 , Санкт-Петербург, ул. Аккуратова, д. 2; e-mail: larisa.chipiga@gmail.com.

\section{УСТАНОВЛЕНИЕ РЕФЕРЕНТНЫХ ДИАГНОСТИЧЕСКИХ УРОВНЕЙ ДЛЯ КТ-ИССЛЕДОВАНИЙ В РОССИЙСКОЙ ФЕДЕРАЦИИ}

\author{
1,2Л. А. Чипига, ${ }^{1}$ И. А. Звонова
}

${ }^{1}$ Санкт-Петербургский научно-исследовательский институт радиационной гигиены им. проф. П. В. Рамзаева, Санкт-Петербург, Россия

2 Национальный медицинский исследовательский центр им. В. А. Алмазова, Санкт-Петербург, Россия

Цель данной работы - оценить перспективы установления референтных диагностических уровней (РДУ) в компьютерной томографии в России. В ходе данной работы собраны данные о протоколах проведения исследований и определены дозы пациентов в России. Анализ распределений доз в разных регионах показал отсутствие значимых расхождений между регионами и возможность установления РДУ в целом по стране. Значения $75 \%$ перцентилей распределений доз предложены в качестве национальных РДУ в России.

\section{THE ESTABLISHMENT OF DIAGNOSTIC REFERENCE LEVELS FOR CT STUDIES IN THE RUSSIAN FEDERATION}

1,2 L. A. Chipiga, ${ }^{1}$ I. A. Zvonova

${ }^{1}$ St. Petersburg Research Institute of Radiation Hygiene name after professor P. V. Ramzaev, St. Petersburg, Russia

${ }^{2}$ National Almazov Medical Research Centre, St. Petersburg, Russia

The aim of the study was to evaluate the possibility of establishing diagnostic reference levels (DRLs) for typical CT examinations in Russia. The study was based on the dose surveys performed in different regions. Comparison between typical dose distributions indicated no significant regional differences, hence allowing establishing DRLs on the national level. The $75 \%$ percentiles of both DLP and effective dose distributions were proposed as the preliminary values of national DRLs.

Цель исследования. Оценить уровни облучения пациентов в компьютерной томографии (KT) в разных регионах России и перспективы установления референтных диагностических уровней (РДУ).

Материалы и методы. В ходе данной работы собраны данные о протоколах проведения исследований в разных регионах России: исследования головы, грудной клетки, брюшной полости, малого таза, всего тела. Исследования с использованием контрастного вещества проводились в несколько фаз, в каждой из которых зона обследования подвергалась облучению. При сборе данных учитывались технические параметры, влияющие на дозу пациентов: режим сканирования, сила тока и напряжение на рентгеновской трубке, время ротации, ширина срезов, значение питч фактора, значения CTDI (компьютерно-томографический индекс дозы) и DLP (произведение дозы на длину сканирования) для более 1200 пациентов. Әффективные дозы рассчитывались согласно МУ 2.6.1.2944-11 [1].

Результаты. Анализ распределений доз показал, что при выполнении исследований головы и грудной клетки различия в дозах незначительны за счет использования фиксированной длины сканирования, которая, как правило, совпадает с размерами анатомической области исследования. Для остальных видов исследований, особенно при проведении исследования в несколько фаз, разброс доз более значителен (до 35 раз). При проведении таких исследований длина сканирования может значительно отличаться в зависимости от телосложения пациента и субъективных предпочтений персонала, а также от поставленной конкретной клинической задачи. 
Высокие уровни облучения пациентов и большой разброс доз при проведении КТ-исследований показывают, что непременным условием широкого внедрения этого метода в медицинскую практику должна стать оптимизации условий его применения, например, путем использования концепции РДУ [2]. Использование этого подхода доказало свою высокую эффективность в зарубежных странах. Анализ распределений доз в двух регионах показал отсутствие значимых расхождений между регионами и возможность установления РДУ в целом по стране. Для установления РДУ в каждом исследовании были выбраны третьи квартили распределения доз для исследований: головы - 1190 мГрхсм (3 мЗв); грудной клетки -500 мГрхсм ( 8 м3в); брюшной полости -780 мГрхсм (12 м3в); малого таза -880 мГрхсм (17 м3в), всего тела 1000 мГрхсм (15 м3в). Для многофазных КТ исследований предлагается использовать соответствующее зоне сканирования значение РДУ для каждой фазы. Значения третьих квартилей распределений доз для объединенной выборки, предложенные в качестве национальных РДУ в Российской Федерации сопоставимы со значениями РДУ в Европе.

\section{ЛИТЕРАTУPA/REFERENCES}

1. Методические указания. Контроль эффективных доз облучения пащиентов при проведении медицинских рентгенологических исследовании (MУ 2.6.1.2944-11). М.: Роспотребнадзор, 2013. [Methodical guidance 2.6.1.244-11. Assessment and accounting of effective dose to the patients undergoing nuclear medicine diagnostic examinations. Moscow: Izdatel'stvo Rospotrebnadzor, 2013 (In Russ.)].

2. Методические рекомендации. Применение референтных диагностиче ских уровней для оптимизации радиационной защиты пациентов в рентгенологических исследованиях общего назначения (МР 2.6.1.0066-12). М.: Роспотребнадзор, 2012. [Methodical recommendations 2.6.1.0066-12. Implementation of diagnostic reference levels to optimize the radiation protection of the patient in conventional radiology. Moscow: Izdatel'stvo Rospotrebnadzor, 2012 (In Russ.)]

\section{Сведения об авторах:}

Чипига Лариса Александровна - младший научный сотрудник лаборатории радиационной гигиены медицинских организаций ФГУН «Санкт-Петербургский научно-исследовательский институт радиационной гигиены им. проф. П. В. Рамзаева» Федеральной службы по надзору в сфере защиты прав потребителей и благополучия человека; 197101, Санкт-Петербург, ул. Мира, д. 8; медицинский физик, ПЭТ-центр, ФГБУ «Национальный медицинский исследовательский центр им. В. А. Алмазова» Минздрава России; 197341 Санкт-Петербург, ул. Аккуратова, д. 2; e-mail: larisa.chipiga@gmail.com;

Звонова Ирина Александровна - доктор технических наук, и.о. заведующего лабора торией радиационной гигиены медицинских организаций ФГУН «Санкт-Петербургский научно-исследовательский институт радиационной гигиены им. проф. П. В. Рамзаева» Федеральной службы по надзору в сфере защиты прав потребителей и благополучия человека; 197101, Санкт-Петербург, ул. Мира, д. 8; e-mail: ir_zv@bk.ru.

\section{РАДИАЦИОННЫЕ РИСКИ ДЕТСКИХ ПАЦИЕНТОВ ОТ РЕНТГЕНОЛОГИЧЕСКИХ ИССЛЕДОВАНИЙ} И. Г. Шацкий

Санкт-Петербургский научно-исследовательский институт радиационной гигиены им. проф. П. В. Рамзаева, Санкт-Петербург, Россия

Радиационный риск оценивался двумя различными способами: на основе поглощенных доз в органах и коэффициентов риска, зависящих от пола и возраста, и путем простого умножения эффективной дозы на номинальный коэффициент риска. Результаты исследования показали, что радиационный риск выше у подростков, чем у детей. Риск для женщин выше в два-четыре раза, чем у мужчин. Упрощенная оценка риска, основанная на эффективной дозе, занижает риск для женщин в 2 раза.

\section{RADIATION RISKS OF CHILDREN PATIENTS FROM X-RAY EXAMINATIONS \\ I. G. Shatskil}

Research Institute of Radiation Hygiene, St. Petersburg, Russia

Radiation risk was assessed in two different ways: based on absorbed doses in organs and sex- and age -dependent risk coefficients, and by simple multiplication of the effective dose by the nominal risk coefficient. The study results demonstrated that the radiation risk is higher in adolescents than in children. The risk for females is higher by a factor of two to four than for males. The simplified risk assessment based on effective dose underestimated risk for females by a factor up to 2 .

Цель исследования. Процесс обоснования использования ионизирующего излучения требует взвешивания клинической выгоды и возможного радиационного ущерба. Существуют упрощенные способь оценки индивидуального риска у пациента, подвергающегося медицинскому облучению, с учетом пола и возраста с использованием эффективной дозы. Более точная оценка пожизненного риска отдаленных стохастических последствий для здоровья пациента достигается при использовании органных доз и половозрастных коэффициентов риска. Целью настоящей работы является оценка пожизненного риска отдаленных стохастических последствий для здоровья различных по полу и возрасту групп детей, подвергающихся различным рентгенографическим исследованиям, с использованием органных доз и половозрастных коэффициентов риска, и последующая его классификация рентгенографических исследований в соответствии с международной шкалой риска

Материалы и методы. В работе использовались данные обследований 33 рентгеновских аппаратов в 29 специализированных детских медицинских организациях России. Для 12 рентгенографических процедур ( рентгенография черепа, органов грудной клетки, шейного отдела позвоночника, грудного отдела позвоночника, поясничного отдела позвоночника - все в двух проекциях - органов брюшной полости и таза - обе в одной проекции) были рассчитаны типичные поглощенные дозы в органах и эффективные дозы пациентов с помощью компьютерной программы РСХМС. Используя полученные органные дозы и половозрастные коэффициенты риска, рассчитанные для российской популяции, были оценены радиационные риски для выбранных исследований для пяти возрастных групп детей со средним возрастом 0, 1, 5 , 10 и 15 лет по модели, приведенной в Публикации 103 МКР3, в соответствии с методикой, опубликованной в статье Иванов и соавт.

Результаты. Установлено, что радиационный риск, обусловленный одним исследованием, имеет выраженную возрастную зависимость для всех анализируемых видов исследований. Для ряда исследований риск больше у подростков. Так, риск при рентгенографии поясничного отдела позвоночника и брюшной полости у детей возраста 10-14 лет в 2-4 раза выше, чем у детей возраста 0-5 лет. При выполнении исследований головы, шейного отдела позвоночника, грудного отдела позвоночника и грудной клетки радиационный риск у женщин существенно выше, чем у мужчин (до семи-восьми раз в отдельных возрастных группах), что объясняется попаданием в поле излучения легких, более радиочувствительных у женщин, чем у мужчин, и молочных желез. При выполнении обследований других органов (поясничного отдела позвоночника, брюшной полости, таз) радиационный риск у женщин и мужчин соизмерим.

Выявлена значительная зависимость радиационного риска от типа и параметров рентгеновского исследования, а также от возраста и пол пациентов.

Выводы. Упрощенная оценка риска, основанная на эффективной дозе для некоторых рентгеновских исследований, приводит к недооценке риска для детей в 4-5 раз, а для подростков - до 2-3.

\section{ЛИТЕРАТУРA/REFERENCES}

1. Балонов М.И., Голиков В.Ю., Кальницкий С.А., Братилова А.А. Риск стохастических эффектов облучения вследствие рентгенографических исследований: зависимость от пола и возраста пациента // Медицинская радиология и радиационная безопасность. 2011. Т. 56, № 4 . C. 71-79. [Balonov M., Golikov V., Kalnitsky S., Bratilova A. Age and sex dependence of the stochastic health effects due to radiography. Med Radiology and Rad Safety. 2011. Vol. 56 (4), pp. 73-79 (In Russ.)]

2. Иванов В.К., Цыб А.Ф., Метлер Ф.А., Меняйло А.Н., Қащеев В.В Радиационные риски медицинского облучения // Радиация и риск. 2011 T. 20, № 2. C. 17-28. [Ivanov V., Tsyb A., Mettler F., Menyaylo A. Kashcheev V. Radiation risks of medical exposure. Radiation and risk, 2011 Vol. 20 (2), pp. 17-28 (In Russ.)]

Сведения об авторе:

Шацкий Илья Геннадьевич - ФБУН «Санкт-Петербургский научно-исследовательский институт радиационной гигиены им. проф. П. В. Рамзаева» Федеральной службы по надзору в сфере защиты прав потребителей и благополучия человека; Санкт-Петербург, ул. Мира, д. 8; e-mail: I.Shatskiy@niirg.ru. 